Service social

\title{
Complexité et interculturel
}

\section{Ghislaine Roy}

Volume 42, numéro 1, 1993

\section{Culture et intervention}

URI : https://id.erudit.org/iderudit/706604ar

DOI : https://doi.org/10.7202/706604ar

Aller au sommaire du numéro

Éditeur(s)

École de service social de l'Université Laval

ISSN

1708-1734 (numérique)

Découvrir la revue

Citer cette note

Roy, G. (1993). Complexité et interculturel. Service social, 42(1), 145-152.

https://doi.org/10.7202/706604ar

\section{Résumé de l'article}

L'auteure se sert de la "pensée complexe " comme support d'un protocole de discussion de cas dans un contexte d'approche interculturelle. L'objectif est l'élaboration d'une grille d'analyse capable de rendre compte de la

multidimensionnalité du « donné social » quand deux porteurs d'identité sont en présence. d'utilisation que vous pouvez consulter en ligne.

https://apropos.erudit.org/fr/usagers/politique-dutilisation/ 


\section{NOTE DE LECTURE}

\section{Complexité et interculturel}

Ghislaine ROY

Travailleuse sociale, SARIMM (Service d'aide aux réfugiés et aux immigrants)

CLSC Parc-Extension

Montréal

À ras de sol, dans l'action tourbillonnaire, dans l'agitation vertigineuse, dans l'opacité de l'existence, il n'est plus de théorie exclusive qui tienne.

Alain Médam

Le présent article a deux points de départ: le malaise que peut ressentir un intervenant social au cours de discussions de "cas » dans un contexte d'approche interculturelle et la découverte d'une notion à mon point de vue essentielle à l'intervenant social, soit la notion de complexité.

II n'est pas question ici d'élaborer une thèse poussée sur la méthodologie de la pensée complexe d'Edgar Morin' ${ }^{\prime}$, bien qu'il puisse être pertinent de le faire éventuellement. Tout au plus, je crois utile de faire part de mes réflexions sur le sujet en titre et $d^{\prime}$ illustrer quelques pistes de travail,

1. Edgar Morin, Introduction à la pensée complexe, Paris, ESF éditeurs, 1990. 
notamment un protocole de discussion de cas. La pensée complexe n'est pas particulière à l'approche interculturelle, mais peut lui être d'un très grand appui.

\section{SITUATIONS-PROBLÈMES: FOUILLIS INEXTRICABLE}

Tous les intervenants sociaux qui ont eu, à un moment donné de leur carrière, I'occasion de présenter un « cas » ou d'assister à la présentation d'un collègue savent ce qu'implique un tel exercice en efforts de systématisation des données, pour que la situation analysée soit la plus claire possible, ou en écoute rigoureuse afin d'offrir des hypothèses d'orientation valables.

Peut-être certains intervenants ont-ils ressenti une impuissance devant la variété des informations présentes, une incapacité de tout retenir, une volonté de mettre de l'ordre dans le fouillis des données, enfin une impression d'avoir à tailler dans le vif pour choisir un aspect du problème au détriment d'un autre.

Souvent, il semble que les grilles de lecture offertes n'appréhendent que partiellement la réalité multiforme des problèmes sociaux. D'où l'impression de découverte ressentie en me familiarisant avec la notion de complexité. Cette notion gagnerait d'ailleurs à faire partie des programmes de cours universitaires, particulièrement les cours de méthodologie de l'intervention en travail social.

\section{MODE DE PENSÉE COMPLEXE}

De quoi s'agit-il, si ce n'est de bâtir une grille d'analyse capable de rendre compte de la multidimensionnalité d'un "donné social», autant dans son incohérence que dans sa forme structurante. Morin dirait: «Il s'agit de s'exercer à une pensée capable de traiter avec le réel, de dialoguer avec lui, de négocier avec lui ${ }^{2}$ ». Au lieu de tenter de maîtriser, d'enfermer toujours plus ce réel, les intervenants sociaux, immergés quotidiennement dans le monde des interrelations, des petits gestes, des petits riens de la vie des autres, pourraient se sentir à l'aise avec le mode de pensée complexe parce qu'intégrateur de ce «social-là » aussi.

Le mot complexité exprime de la confusion, de l'embarras, de l'incapacité à définir de façon simple, à mettre de l'ordre. Pensons à l'intervenant social qui essaie de présenter son « cas » le plus simplement possible, mais qui n'y arrive pas, tant est grande l'abondance des détails. Cette confusion devant

2. Id., p. 10. 
l'exubérance des petits faits humains lui donne d'ailleurs beaucoup de fil à retordre dans la systématisation d'informations souvent contradictoires à partir desquelles il construit des « histoires sociales » cohérentes.

C'est un défi : le défi du complexe qui stimule une nouvelle approche de la réalité. Depuis toujours, le mode d'organisation du savoir semble plutôt basé sur l'abstraction, la disjonction, la réduction. La pratique professionnelle des travailleurs sociaux ou les approches utilisées en travail social ne font pas exception.

Tout en admettant la nécessité d'une gestion efficace des problématiques sociales, ne faudrait-il pas garder en tête la globalité et la complexité des situations-problèmes vécues par la clientèle des services sociaux? Voilà une préoccupation difficile à retenir dans un quotidien de pratique professionnelle. C'est pourquoi il est nécessaire d'arrimer le «ras-du-sol » de nos interventions à une réflexion théorique et méthodologique, ainsi que le permet une notion comme celle de la complexité.

\section{MÉTHODOLOGIE COMPLEXE}

Est-ce à dire que la complexité n'admet que l'absence d'ordre? Au contraire, le fait d'intégrer la notion de complexité aux grilles d'analyse dont nous nous servons habituellement en tant qu'intervenants sociaux exige une recherche rigoureuse en matière de renouvellement d'outils de travail, de grilles de données. La complexité nécessite d'ailleurs une stratégie organisée dès que survient l'inattendu, l'incertain. Stratégie qui n'essaie pas cependant de tout programmer. Elle admet que la vie, que le réel ne pourra jamais se laisser enfermer dans une grille, si perfectionnée soit-elle!

Le réel ne cesse de bouger. En tant qu'intervenants sociaux, nous essayons de le circonscrire, de l'encadrer, de le planifier. Au lieu de cela, il faudrait « accepter le disparate, le non-unifié, le non-unifiable, le non-réductible à un seul point de vue» $\left(\right.$ Médam $\left.^{3}\right)$. Accepter de partir de la réalité complexe pour construire des instruments de travail. "Donner à voir » les choses de la vie, sorte de fouillis inextricable, tout en les classant: mouvement de va-et-vient entre la position du «renifleur social» attentif à l'instituant, au souterrain et celle du «taxinomique » qui classe les situations instituées, officielles (Maffesoli $\left.{ }^{4}\right)$.

Pour ce faire, il faut intégrer des notions appartenant à différentes disciplines intellectuelles, comme l'anthropologie, la sociologie, la psychologie,

3. A. Médam, "Des grilles et des vies», RIAC, 15/55, 1986, p. 153.

4. M. Maffesoli, La connaissance ordinaire. Paris, Librairie des Méridiens, 1985, p. 17. 
voire la philosophie. Sortir de l'orthodoxie de nos champs disciplinaires et nous rencontrer dans les marges de plusieurs disciplines. De là peut surgir la créativité.

Il faut également apprivoiser, pour le compte de la pratique, le concept de praticien-chercheur ou celui qui est «dedans » tout en s'exerçant à avoir le « regard éloigné ». N'est-ce pas la position de l'intervenant social qui participe au quotidien de ses "clients", auquel il doit donner un sens logique?

Toujours dans un effort pour élaborer une méthode d'intervention complexe, il nous faudrait peut-être, comme intervenants, créer des rapports de solidarité avec nos «clients» en utilisant, notamment, la multiplicité d'appartenances que nous possédons (nationale, professionnelle, de classe sociale, de rattachement institutionnel...). Mutiplicité d'appartenances pouvant devenir occasions de rencontres avec l' «autre», de création d'un lien de sujet à sujet. Dans cette conception, $I^{\prime}$ « autre » devient un partenaire sans lequel l'intervenant est impuissant à rendre compte de la réalité complexe de ce qui lui est apporté.

\section{CONTEXTE INTERCULTUREL}

Depuis quelques années se sont développées des théories, des notions de base, des réflexions fort pertinentes dans une perspective de travail social en contexte interculturel. Des efforts de systématisation des pratiques interculturelles ou d'approches interculturelles ${ }^{5}$ ont été entrepris.

II n'en demeure pas moins que l'effort le plus grand à fournir pour intégrer ces nouvelles notions (que l'on pense aux notions d'identité, de stratégies identitaires, de migration, de culture, d'interaction, de chocs culturels, etc.) sur le plan du discours et des approches respectives revient aux intervenants sociaux eux-mêmes.

Comment rendre compte, sous forme d'instruments de travail, de I'intervention en contexte interculturel, tout en considérant la complexité et l'appartenance de désordres inhérents à toute situation-problème?

En ce qui regarde, par exemple, l'étape du processus d'évaluation dans le plan d'intervention, il n'y a d'autre choix que de créer un nouvel outil de travail. Parmi les points à retenir, dans une grille d'évaluation renouvelée, dont

5. Pour ne citer que quelques documents à partir desquels il est possible au lecteur de prendre connaissance d'une multitude de références : Chiasson-Lavoie et al., L'approche interculturelle auprès de réfugiés et de nouveaux immigrants, CSSMM, 1991 ; G. Legault et M. Lafrenière, Femmes, migrations, interventions: une rencontre interculturelle, Université de Montréal, 1992 ; J. Bertot et A. Jacob, Intervenir avec les immigrants et les réfugiés, éd. du Méridien, 1991 ; G. Roy, "Incompréhensions interculturelles et ajustements de pratiques chez les travailleurs sociaux», Revue canadienne de service social, vol. 8, n 2, été 1991. 
le but serait d'intégrer les dimensions psychosociales de la migration tout en fournissant un cadre complexe d'analyse, relevons les éléments suivants : le contexte migratoire (départ, arrivée, projet de retour), le mode de vie dans le pays d'origine, la situation-problème telle qu'elle est perçue par les parties en présence, la connaissance et l'utilisation du groupe ethnique référentiel, le repérage de situations fragilisantes, le réseau $d^{\prime}$ appartenance ${ }^{6}$.

Cette façon d'évaluer une situation-problème tente $d^{\prime}$ 'intégrer dans I'analyse une variété d'éléments qui vont bien au-delà de la demande exprimée. C'est un processus d'évaluation basé sur des hypothèses spécifiques concernant la migration, l'intervention entre deux porteurs d'identité et enfin les aires culturelles respectives de l'intervenant et du «client ». Bref, le processus d'évaluation d'une situation donnée étant au cœur des activités professionnelles des intervenants sociaux, il doit, par le fait même, traduire le plus fidèlement possible la complexité des histoires de vie qui sont relatées aux intervenants.

Il a été question plus haut de discussions de «cas » et du malaise que des intervenants sociaux peuvent ressentir quand, notamment, ils ont à privilégier un aspect d'une situation-problème aux dépens d'autres aspects. N'y aurait-il pas moyen de parer au danger de la «myopie culturelle et institutionnelle » en développant des instruments de travail qui rendraient compte de la complexité des situations-problèmes? Déjà, dans une communication donnée aux journées d'étude du Conseil consultatif du personnel clinique, à Montréal, il avait été question de la construction d'un "protocole de discussion de cas » permettant de révéler des dimensions majeures, complexes, inhabituelles, sur le thème de la parenté élargie ${ }^{7}$.

Je veux reprendre ici, sous forme de proposition, l'élaboration d'un «protocole de discussion de cas » qui vise à illustrer une situation-problème, en posant d'abord la complexité de l'ensemble des données. Ce schéma de protocole se base sur la définition de «la différence» dans la perspective interactionniste $\mathrm{d}^{\prime}$ Abdallah-Pretceille ${ }^{8}$ qui est: « (...) un rapport dynamique entre deux entités qui se donnent mutuellement un sens».

Le protocole de discussion de cas présenté ci-après se base également sur la théorie de la communication où il est notamment question d'émetteur,

6. Cette grille d'évaluation dans une approche interculturelle est publiée dans les annexes des documents de Chiasson-Lavoie et al. ainsi que de G. Legault et M. Lafrenière, déjà cités.

7. Quelques communications dont celles portant sur le protocole de discussion de cas et la notion de parenté ont été publiées dans I'Écho professionnel, vol. 5, no 3, sept.-oct. 1992.

8. M. Abdallah-Pretceille, "Pédagogie interculturelle: bilan et perspectives", dans L'Interculturel en éducation et en sciences humaines, tome 1, Le Mirail, 1985, p. 31. 


\section{Proposition de protocole de discussion de cas}

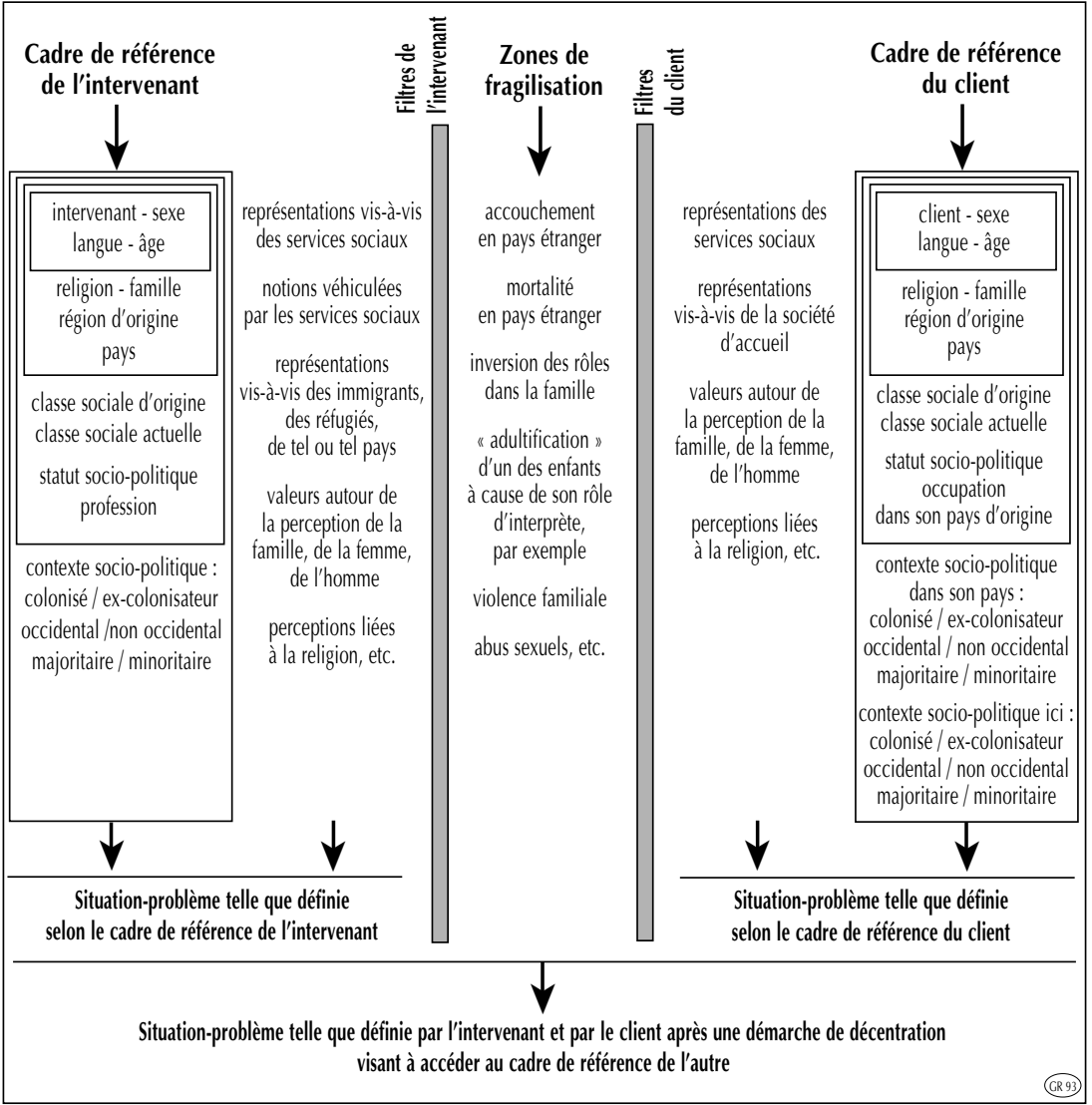

de récepteur, de message, de codage, de décodage et de feed-back ${ }^{9}$. En communication interculturelle, ce n'est pas seulement le message qui fait l'objet d'attention, mais aussi les locuteurs eux-mêmes, avec leurs valeurs et leurs contextes respectifs.

Le protocole s'appuie enfin sur la conception de Cohen-Emerique ${ }^{10}$ relativement à l'observation de signaux culturels différenciateurs, de filtres et

9. Le lecteur trouvera ces notions approfondies dans R. Bourque, Les relations interculturelles dans les services sociaux, guide d'animation, 1989.

10. M. Cohen-Emerique, «Chocs culturels et relations interculturelles dans la pratique des travailleurs sociaux», Cahiers de sociologie économique et culturelle, décembre 1984 ; «Travailleurs sociaux et migrants. La reconnaissance identitaire dans le processus d'aide», Chocs de cultures, L'Harmattan, 1989 ; "Le modèle individualiste de sujet», Cahiers de sociologie économique et culturelle, juin 1990. 
d'écrans sociaux, de distorsions dans l'attribution de sens dans le processus d'évaluation, de même que des zones de fragilisation porteuses d'éléments déclencheurs de crises.

Voici comment un intervenant social soucieux de tenir compte de la complexité du réel avec lequel il doit travailler, en même temps que préoccupé d'offrir à ses collègues à qui il présente un «cas » un réel non amputé de ses éléments multiples, pourrait faire.

À l'aide de cette grille, il présenterait la situation-problème dans ses multiples dimensions: son propre cadre de référence, ses filtres, ses définitions du problème, l'identification des zones porteuses d'éléments déclencheurs de crises (zones de fragilisation), le cadre de référence du «client», les filtres du «client», sa définition du problème, les zones fragiles telles que définies par le «client». Tout cela, afin d'arriver à une définition complexe d'un problème qui serait le résultat d'une démarche de décentration et de pénétration du cadre de référence de l'autre.

Voici comment un intervenant social pourrait amorcer une discussion de «cas», en proposant d'abord une sorte de portrait constitutif d'une multiplicité d'éléments.

Il est évident que ce qui est inscrit dans les colonnes «filtres » et «zones de fragilisation » constitue des exemples et non des éléments définitifs. II revient à chaque intervenant, selon la situation-problème à laquelle il fait face, d'inscrire à l'intérieur de ces repères ce qui est pertinent pour la compréhension du problème et pour la fidélité à un mode de pensée complexe.

Il faudrait davantage que quelques pages pour expliciter ce protocole qui tente à sa façon d'illustrer la complexité du jeu interculturel dans une relation entre professionnels et « clients ». Toutefois, ce protocole veut essayer de rendre opérationnalisable un élément clé dans l'approche interculturelle, à savoir l'intervenant lui-même, comme porteur d'identité. En effet, si I'hypothèse de l'influence identitaire de l'intervenant est retenue, il faut lui accorder la place qu'elle mérite. C'est ce à quoi ce protocole s'attache, entre autres objectifs, dont celui d'adapter la pratique professionnelle des intervenants sociaux à une théorie de l'intervention en contexte interculturel.

\section{LE DÉSORDRE COMME PRINCIPE VITAL DYNAMIQUE}

«Penser complexe, c'est se préparer à l'inattendu, c'est secouer notre paresse d'esprit, c'est sortir des déterminismes » $\left(\right.$ Morin $\left.{ }^{11}\right)$. C'est créer, c'est inventer. La pensée complexe ne repose pas sur l'ordre ou sur la clarté, et elle les sait insuffisants. C'est pourquoi il faut créer, dans l'intervention en contexte

11. E. Morin, op. cit., p. 111. 
interculturel comme dans d'autres contextes d'ailleurs, de nouveaux outils de travail basés sur un mode complexe d'appréhension des réalités sociales.

Les grilles de lecture fournies par les modèles socio-empiriques sont impuissantes à rendre compte de la complexité des situations-problèmes dans un contexte interculturel. II faut remettre en question les modèles traditionnels et en construire de nouveaux.

Il faut ainsi rendre compte, le mieux possible, à la fois de l'incohérence, de la polysémie du «donné social » et de ses formes structurantes. Le «donné social » est fourni quotidiennement aux intervenants sociaux par l'entremise des situations-problèmes auxquelles font face leurs «clients »: «(...) dire d'une manière cahotante et inachevée la vie sociale en son déroulement, dans ses détours et ses arrêts » (Maffesoli ${ }^{2}$ ). Voilà l'essentiel, le spécifique du travail des intervenants sociaux en contexte interculturel et autres contextes sociaux.

Les intervenants sociaux sont des témoins privilégiés des faits de l'existence humaine qu'ils doivent «traduire » dans une forme compréhensible pour la machine bureaucratique.

C'est une position complexe que celle des intervenants sociaux qui effectuent un va-et-vient continuel entre l'espace d'intersubjectivités qu'ils créent et l'espace défini par leur rôle professionnel. Position complexe qui fait des intervenants sociaux des acteurs paradoxaux devant se construire des instruments complexes d'appréhension des réalités sociales organiquement constituées d'ordre et de désordre.

12. M. Maffesoli, op. cit., p. 76. 